\title{
Mapping QTLs Related to Plant Height and Root Development of Eragrostis tef under Drought
}

\author{
Degu Hewan Demissie (Corresponding author) \\ Graduate School of Life and Environmental Sciences, University of Tsukuba \\ Tsukuba, 305-8572, Japan \\ Tel: 81-90-61-473-711Ｅ-mail: Hewan.dd@gmail.com \\ Tatsuhito Fujimura \\ Graduate School of Life and Environmental Sciences, University of Tsukuba \\ Tsukuba, 305-8572, Japan \\ Tel: 81-90-61-473-711Ｅ-mail: tfujimura@sakura.cc.tsukuba.ac.jp
}

\begin{abstract}
The effects of water-stress on root and shoot growth of tef (Eragrostis tef) was evaluated with a population of 94 recombinant inbred lines (RILs) derived from a cross between tef (cv. Kaye Murri) and E. pilosa. The young seedlings were cultured under well-watered (soil water potential; $0.2 \mathrm{MPa}$ ) and water-stressed (-1.6 MPa) conditions, and plant height and primary root length were measured after 15 days of culture. Kaye Murri consistently showed larger plant height and longer primary roots than E. pilosa under drought. Quantitative trait loci (QTLs) were also mapped in relation to water-stress using traits of RILs. Five and seven QTLs for plant height and nine and eight QTLs for primary root length under both of well-watered and water-stressed conditions were identified, respectively. Seven and six QTLs for indexes of response to plant height and primary root length were also found, respectively. Phenotypic variations for a single QTLs was in the range of $2 \%$ to $20 \%$. Beside of Kaye Murri, E. pilosa provided promotive QTLs for development of shoots and roots under drought, indicating this species is also an important genetic resource for the breeding of tef.
\end{abstract}

Keywords: Tef, Eragrostistef, E. pilosa, Primary root, Quantitative trait loci (QTLs), Recombinant inbred lines (RILs)

Abbreviations: LG, linkage group; PH, plant height; PRL, primary root length; PHC, plant height under well-watered conditions; PHS, plant height under water-stressed conditions; PRLS, primary root length under stress; PRLC, primary root length under well-watered conditions; QTLs, quantitative trait loci; RILs, recombinant inbreed lines.

\section{Introduction}

Tef (Eragrostis tef) is an important staple crop grown in Ethiopia where soil conditions range from flooded to drought (Seyfu, 1993). Tef is an allotetraploid with an estimated genome size of $730 \mathrm{Mbp}$ and $2 \mathrm{n}=4 x=40$ chromosomes. It is a C4-metabolism cereal plant of medium stature and has a short growth-duration period (Jones et al., 1978; Berhe et al., 1989, Tavasoli, 1986; Ayele et al., 1996). It belongs to the grass family Poaceae, subfamily Chloridoideae. It is the only cereal crop member of the tribe Eragrostidae and genus Eragrostis, which contains about 350 species (Watson and Dallwitz, 1992). It is grown as a major cereal grain in Ethiopia and currently it occupied 2 million hectares, which accounts for $28 \%$ of the cereal crops grown in the country (Central Statistical Authority, 2006). The grain is used to make a variety of food products, including injera, a spongy fermented flat bread that serves as the staple food for most Ethiopians.

Plant heights are important traits that directly contribute to grain productivity in tef (Yu et al., 2007). However lodging is the major constraint to increasing the yield of tef (Seyfu, 1997). In addition, when optimum amount of fertilizer is applied to increase the yield, high incidence of lodging occurs. As a consequence, the yield from the crop is severely reduced in terms of total grain yield and quality. The lodged plant also poses a great problem for harvesting. Dwarf rice and wheat varieties were developed by classical plant breeding methods, contributing to the green revolution in the 1960s. Higher yields were obtained from these dwarf crops because their short stature 
reduced lodging (Peng et al., 1999). However improving lodging resistance of cereals by focusing only the plant height might have negative effect if precautions are not taken by considering other factors such as drought stress.

Even though tef is highly adapted to drought, water deficit is one of the main limiting factors of tef production. The early seedling stage of this crop is the most sensitive to water stresses (Seyfu, 1993 and Degu et al, 2009). A deep root system has been thought to be advantageous for improved drought tolerance in many cereal crop plants (O'Toole, 1982; Fukai and Cooper, 1995). Under well-watered conditions, root system development was observed to have a positive effect on subsequent plant growth during progressive water stress (Hoque and Kobata, 1998; Azhiri-Sigari et al., 2002; Kamoshita et al., 2002). Azhiri-Sigari et al. (2002) and Kamoshita et al. (2002) demonstrated genotypic variation in constitutive root traits, and subsequent responses of adaptive root traits, especially in deeper soil layers. Greater root elongation in deeper soil layer resulted in improved water extraction. Improved seedling vigor was also valuable to growth afterward (Kondo et al., 2003 and Cui et al., 2008).

Patterns of root development are also diversified among tef cultivars (Ayele and Nguyen, 2001) and traits such as root length and plant height also diversified significantly. A particular cultivar of tef, Kaye Murri, exhibits an elongation of primary roots under water-stressed conditions compared to E. pilosa (Degu et al., 2008). And regulation of root development is proposed to be one of the adaptive mechanisms to water-stress of tef. And $E$. pilosa has contributed useful breeding traits, such as earliness and short stature (Tefera et al., 2003). Therefore, utilization of E. pilosa as a donor in an inter-specific cross is a useful strategy for broadening the genetic diversity of plant height and root traits in cultivated tef.

Modification of the tef root system by conventional breeding is not easy because of tedious screening techniques and the plastic nature of the root system. The application of molecular markers greatly simplifies the screening for traits that are difficult to measure and to induce in nature (O'Toole, 1989; Hanson et al., 1990; Tanksley, 1993). High-density linkage maps constructed in major crops enabled the dissection for the genetic basis underlying complex traits into their individual components (Lander and Botstein, 1989; Tanksley, 1993). Recently, molecular genetic maps of tef were also constructed by Zhang et al. (2001) and Yu et al. (2006), and made it possible to identify and locate genes or quantitative trait loci (QTLs) for yield and yield-related traits (Yu et al., 2007). This confirms that DNA marker-assisted selection could be an alternative to conventional screening. For this to succeed, basic information, QTLs by environment interaction, consistency, and differences due to genetic background and epistasis need to be obtained.

The objective of this study was to identify QTLs for plant height and primary root in order to determine the relationship between plant height and root length, at low and high soil moisture conditions between $E$. tef and E. pilosa with recombinant inbreed lines, at seedling stages.

\section{Materials and methods}

\subsection{Plant material and growth environment}

A population of recombinant inbred lines (RILs) was used in this study. They were the F9th generation of the progeny derived from an interspecific cross between E.tef (cv. Kaye Murri) and E. pilosa (accession 30-5). This population was developed at the Debre Zeit Agricultural Research Center in Ethiopia (Tefera et al., 2003).

Our previous screening experiments revealed that the elongation of primary root of Kaye Murri was stimulated by water deficit much longer than that of E. pilosa (Degu et al., 2008). Based on these screening results, experiments were performed using two water supply conditions. Plants were cultured under well-watered (-0.03 $\mathrm{MPa})$ and water-stressed (-1.6 MPa) conditions in a growth chamber which was maintained as follows: mean day and night temperatures were at 28 and $20{ }^{\circ} \mathrm{C}$, respectively; light regime was $860 \mu \mathrm{E} \mathrm{m} \mathrm{m}^{-2} \mathrm{~s}^{-1}$ over a $14 \mathrm{~h}$ photoperiod; and the relative humidity was at $65 \% \pm 5 \%$. A soil, a sand-loam-peat mixture (Soil for Flowers and Vegetables, Kato, Co. Ltd., Japan), was used after sterilization. The plants were grown in a plastic box (30 cm height, $25 \mathrm{~cm}$ width and $1.2 \mathrm{~cm}$ thickness) lined with a polyethylene sheet to facilitate the analysis of root development. The box was surrounded by a rectangular wood frame (about $6 \mathrm{~mm}$ in thicknesses) to provide support and to prevent light penetration. Positions of the plastic boxes were frequently rotated in a randomized design.

To set identical soil moisture conditions, the soil was initially oven-dried for 48 hours at $110{ }^{\circ} \mathrm{C}$. After filled with soil, the boxes were dipped in water to wet the soil via capillary action and then to establish well-watered condition. Water-stressed conditions were established by mixing the oven-dried soil with the soil containing a determined moisture condition. The soil was left for three days and some soil was added if the soil level was depressed. The boxes were left for another two days before planting. No further depression was observed during 
the growth of seedlings. Just before planting, $200 \mathrm{ml}$ of water was added to the soil to establish seedlings. After this initial watering, no additional water was added to the box. At the end of the experiment, soil water potential was measured with a dew point micro-voltmeter (WESCOR HR33-T) and moisture content of the soil was measured by weight (Table 1).

Plants of the 94 RILs and their parents were harvested after 15 days of culture and plant height (PH) and primary root length (PRL) were measured. Random complete block design implemented (factorial of 2 watering regimes x 96 plant types). Ten plants in one box constituted one experimental unit and the average value of 10 plants with 10 replications was used in the statistical analysis.

\subsection{Map construction, statistical analysis and QTL analysis}

A molecular map was constructed with this RILs population and 192 loci derived from 142 molecular markers, representing 21 linkage groups and constituting $2081.5 \mathrm{cM}$ in a total distance (Yu et al., 2006). The mean distance between two markers was $12.3 \mathrm{cM}$.

Genetic variations for all measured traits were estimated with GenStat (Lawes Agricultural Trust). Broad sense heritability at the mean genotypic level, $h^{2}$, was computed as:

$$
h^{2}=\delta^{2} \mathrm{G} /\left[\delta^{2} \mathrm{G}+\left(\delta^{2} \mathrm{e} / n\right)\right]
$$

Where, $\delta^{2} \mathrm{G}$ and $\delta^{2} \mathrm{e}$ are the estimate of genetic and residual variances, respectively, derived from the expected mean square of the analysis of variance, and $n$ is the number of replications.

Quantitative trait loci (QTLs) analysis was performed using QTL Cartographer with Windows QTL Cartographer ver. 2.5 (Wang et al., 2006). The CIM analysis used forward regression with a walking speed of 2 $\mathrm{cM}$ and a window size of $10 \mathrm{cM}$ Kosambi function. To identify an accurate significance threshold for the trait, an empirical threshold was determined for CIM 1000 permutations across all 21 linkage group. A QTL was considered stable if it was detected in SMA, IM and CIM.

Epistasis in the parameters was analyzed using the program QTL mapper (Wang et al., 1999). In addition, the additive effect and percentage of variation explained by an individual QTL were also estimated.

\section{Results}

\subsection{Plant response to drought stress}

After 15 days of culture under well-watered and water-stressed conditions, PH and PRL of the plants were measured (Fig.1). There was a significant reduction of PH from plants of parents and RILs under water-stressed condition; however, PRL was increased under this condition $(P \leq 0.01)$. PH under well-watered (PHC) and water-stressed (PHS) conditions ranged from 2.6 to $24.3 \mathrm{~cm}$ and 1.3 to $19.3 \mathrm{~cm}$, respectively. PRL ranged 3.0 to $23.0 \mathrm{~cm}$ and 3.4 to $32.3 \mathrm{~cm}$ under well-watered (PRLC) and water-stressed (PRLS) conditions, respectively. Most of the traits studied here with the RILs were normally distributed.

Under water-stressed conditions, mean PH of RILs were between those of Kaye Murri and E. pilosa (Fig. 1). The mean of PH of the RILs under well-water condition is less than that of Kaye Murri. The mean of PRL of the RILs under well-watered conditions was higher than that of Kaye Murri, however, under water-stressed conditions, the mean values of PRL fell bellow the two parental lines. The PH and PRL under well-watered and water-stressed conditions demonstrated a much higher heritable component of more than 60\% (Fig. 1).

Although the significant level is not so high, negative correlations were found between means of PH and PRL of the RILs under well-watered and water-stressed (Table 2) conditions, respectively.

\subsection{QTLs analysis}

QTLs for PH and PRL under two water conditions are presented in Table 3 and Fig. 2 with the flanking markers, linkage groups carrying the QTLs, the sign of the QTLs effect and their coefficients of determination $\left(\mathrm{R}^{2}\right)$. The $\mathrm{R}^{2}$ value represents the proportion of the phenotypic variation of the trait explained by allelic substitution at the given molecular marker locus.

Five and seven QTLs were identified for PHC and PHS, respectively. QTLs linked to TCD99b/CNLT119 and RZ154 contributed the highest phenotypic variations for PHC and PHS, respectively. The contribution of the QTLs to the total variations in PHC and PHS were 20\% and 43.5\%, respectively. Kaye Murri contributed the majority of traits for PHC and PHS, respectively.

Nine and eight QTLs were identified for both PRLC and PRLS, respectively. QTLs linked to CNLT49b contributed the highest (16\%) of the total variation of PRLC. While QTLs linked to ISSR549a/CSU38 explained 
9\% of the total variation of PRLS. Both Kaye Murri and E. pilosa contributed the positive phenotypic effect for the two traits.

A QTLs map was also generated from the difference in PH (PHD) and in PRLs (PRLD) between the stressed and control plants (Fig. 2). QTL linked to RZ154/TCD230a contributed 19\% phenotypic variations for PHD. While two QTLs linked to ISSR548b/RM170 and RZ214b explained from $10 \%$ of phenotypic variations for PRLD. Both Kaye Murri and E. pilosa contributed the additive effects.

Two pairs for PHC and PHS, and one pair for PRLC and PRLS of epistatic loci were identified (Table 4). Among them, some combinations showed strong epistasis, 18 to $20 \%$ of the total variations.

\section{Discussion}

The parental lines investigated in this study had already been shown to have genetic variability for root morphological traits (Degu et al., 2008). In the present study, plant height and root length were investigated to establish differences among genotypes under water stressed conditions. The plant height and primary root length are the most reliable traits for establishing differences among the genotypes due to high $h^{2}$ (Fig. 1). Most of the Plants subjected to water-stress decreased in PH and increased in PRL that were clearly shown in this study (Fig. 2). Negative correlations were also observed between PH and PRL of the RILs (Table 2). These negative correlations were supposed to be an adaptive morphological growth response to water-stress of tef. This indicates that roots may continue to grow under water deficit, which inhibits plant height growth as mentioned by $\mathrm{Wu}$ and Cosgrove (2000). Such morphological responses to water-stress have also been reported in many other plant species such as Ziziphus (Amdt et al., 2001), Populus (Marron et al., 2002), maize (Vamerali et al., 2003), pearl millet (Kusaka et al., 2005) and wheat (Whalley et al., 2005). And the present experiment is also in agreement with our previous work (Degu et al., 2008). Under this response, we analyzed QTLs for these morphological changes under water-stress.

The CIM, IM and SMA methods were concordant in the identification of the QTLs. However the values of phenotypic variance by IM analysis were lower than by CIM analysis, indicating that the latter is more effective at estimating QTL effects.

Many QTLs with high LOD values but with the small additive values were observed during the course of QTLs analysis, although we cannot exclude the possibility that a major QTL is hiding in the approximately $12 \%$ of the tef genome that was not covered in this study. The size of the individual QTL effects is difficult to assess after this initial genome scan for several reasons. It is likely that some estimated QTL effects have been inflated since a common problem in QTL studies is that those loci where the effect by chance is overestimated are more likely to reach statistical significance (Mackinnon and Georges, 1992; Göring et al. 2001). It is also possible that individual QTL effects have been overestimated because they represent effect of two or more linked QTLs, each with a smaller individual effect. For example PHC has one QTL on LG 1 with LOD value 5.4; however the relatively large phenotypic variations compared to the QTL identified on LG 17 with LOD value of 16.6 the other QTLs can be explained because of segregation distortion at these loci (Yu et al., 2007). Furthermore, it is possible that some QTL effects have been underestimated because of statistical analysis of the population. Another possible bias when estimating QTL effects may be caused by the fact that a distortion of the segregation lines may also diminish the estimated effects (Yu et al., 2007). Our finding of QTLs, each with small individual effects, is in good agreement with the results of QTL studies of tef (Yu et al., 2007).

As most of the QTLs expressed under well- watered conditions were not expressed under drought conditions (Table 3), regulations of shoot and root development under these conditions may be very complex and via a different path in tef. Promotive QTLs for all measured traits were conferred from both parents as shown in Table 3. E. pilosa may also contain a promotive gene for PH and PRL development responding to drought, although the PH and PRL of E. pilosa are smaller than Kaye Murri.

Coincident QTLs for traits associated with PHD, PRLC and PRLS have been identified on LGs 6 which is linked to marker CNLT49b.The positive weight values of the collocating QTLs suggest two possible interpretations. Allelic variation at a single locus could be responsible for the concurrent increase (or decrease) of phenotypic values for the relevant traits. Alternatively, alleles from the two parent's effect may be present at different loci. In either case, selection for a single set of linked markers would lead to an increase for each trait.

We also analyzed PHD and PRLD, which are used as indexes of response of PH and PRL to drought (Table 3). QTL linked to RZ154/TCD230a contributed the highest phenotypic variation (20\%) indicating the importance of this locus for the regulation of plant height. However many of the QTLs detected under well-watered and waterstressed conditions were not detected for the index. Such discrepancies might be raised because E. pilosa has less 
ability than Kaye Murri to modify PRL in response to water-stress (Table 3); the former may have potent regulatory genes of PRL under water stress as shown in QTLs for PRLD. From these five and two QTLs were contributed from E. pilosa for PHD and PRLD, respectively. Indicating E. pilosa, a weedy species of tef, is an important genetic resource for the breeding of tef. This suggests a complex picture of the genetics behind the responses of plant height and root length to the effect of water- stress of tef.

QTLs from the six traits are located on LG 6 (Fig. 2). This indicates the QTL locus between CNLT49b and RM176 may be very important in controlling many traits of tef since it accounts up to $16 \%$ of the phenotypic variance. This locus has also been reported as the QTL for yield in tef (Yu et al., 2007) and its importance must be emphasized for the breeding of tef.

Further more, multiple regression analysis and interaction of two markers for each trait showed epistatic interaction (Table 4) accounting for a total of $16 \%$ to $20 \%$ of phenotypic variance. The most interesting interaction among those combinations was between TCD95 and RM170b for PRLC and RM170b and CDO38 for PRLS. These QTLs were also shown to be linked to grain yield (Yu et al., 2007). Interestingly TCD95 participated in four kinds of combinations, which alone was not effective. This indicates that it is possible that the gene lying around this locus may be an important gene controlling diversified activities of tef.

In conclusion integrated approaches are necessary to identify the genetic and molecular mechanisms that underlie complex traits of tef under water-stress. To achieve that goal, quantitative genetic methods such as QTLs analysis have to be combined with molecular plant physiology. Our study furnished reliable QTLs data for early plant height and primary root length under water stress conditions of tef. These results could be used as a basis for further investigations to characterize the major QTLs at the molecular level. QTLs to be identified in those studies may serve as a starting point for retrieving natural allelic variation to improve tef plants for drought tolerance.

\section{Acknowledgements}

The authors would like to thank The Ministry of Education of Japan for supporting the study of tef via a Japanese Ministry of Education Scholarship. We would also like to offer special thanks to the staff at the Plant Genetic Engineering Laboratory, Tsukuba University, Japan. The authors would also like to express special thanks to Dr. Yusaku Uga (National Institue of Agrobiological Sciences) for giving us comments and suggestions to our QTLs analysis.

\section{References}

Amdt, S.K., Clifford, S.C., Wanek, W., Jones, H.G., Popp, M. (2001). Physiological and morphological adaptations of the fruit tree Ziziphus rotundifolia in response to progressive drought stress. Tree Physiol., 21, 705-715.

Ayele, M., Dolezel, J., Van, D.M., Brunner, H., Zapata-Arias, F.J. (1996). Flow cytometric analysis of the nuclear genome of the Ethiopian cereal tef [Eragrostistef(Zucc.) Trotter]. Genetica, 98, 211-215.

Ayele, M., Nguyen, T.H. (2001). Diversity for osmotic adjustment and root depth in tef [Eragrostis tef (Zucc) Trotter]. Euphytica, 121, 237-249.

Azhiri-Sigari, T., Yamauchi, A., Kamoshita, A., Wade, L.J. (2000). Genotypic variation in response of rainfed lowland rice to drought and rewatering II. Root growth. Plant Prod. Sci., 3, 180-188.

Berhe, T., Nelson, L.A., Morris, M.R., Schmidt, J.W. (1989). Inheritance of phenotypic traits in teff III. Panicle form. J. Hered., 80, 67-70.

Central Statistical Authority. (2006). Demographic and Health Survey of Ethiopia. Addis Ababa, Ethiopia and Calverton, Maryland, USA.

Cui, K., Huang, J., Xing, Y., Yu, S., Xu, C., Peng, S. (2008). Mapping QTLs for seedling characteristics under different water supply conditions in rice (Oryza sativa). Physiol. Plant.,132,1-53.

Degu, H.D., Ohta, M., Fujimura, T. (2008). Drought tolerance of Eragrostis tef and development of roots. Int. J. Plant Sci., 169, 768-775.

Fukai, S., Cooper, M. (1995). Development of drought-resistant cultivars using physio-morphological traits in rice. Field Crops Res., 40, 67-86.

Göring, H.H., Terwilliger, J.D., Blangero, J. (2001). Large upward bias in estimation of locus-specific effects from genome wide scans. Am. J. H. Genetics, 69, 1357-1369. 
Hanson, A.D., Peacock, W.J., Evans, L.T., Arntzen, C.J., Khush, G.S. (1990). Drought resistance in rice. Nature, 345, 26-27.

Hoque, M.M., Kobata, T. (1998). Growth responses of drought-resistant rice cultivars to soil compaction under irrigated and succeeding non-irrigated conditions during the vegetative stage. Plant Prod. Sci., 1,183-190.

Jones, M.G., Ponti, J., Tavassoli, A., Dixon, P.A. (1978). Relationships of the Ethiopian cereal tef [Eragrostis tef (Zucc.) Trotter]: evidence from morphology and chromosome number. Ann. Bot., 42,1369-1373.

Kamoshita, A., Zhang, J., Siopongco, J., Sarkarung, S., Nguyen, H.T., Wade, L.J. (2002). Effects of phenotyping environment on identification of QTL for rice root morphology under anaerobic conditions. Crop Sci., 42, 255-265.

Kondo, M., Pablico, P.P., Aragones, D.V., Agbisit, R., Abe, J., Morita, S., Courtois, B. (2003). Genotypic and environmental variations in root morphology in rice genotypes under upland field conditions. In: J. Abe, Editor, Roots, pp. 189-200.

Kusaka, M., Lalusin, A.G., Fujimura, T. (2005). The maintenance of growth and turgor in pearl millet (Pennisetumglaucum [L] Leeke) cultivars with different root structures and osmo-regulation under drought stress. Plant Sci., 168, 1-14.

Lander, E.S., Botstein, D. (1989). Mapping Mendelian factors underlying quantitative traits using RFLP linkage maps. Genetics, 121, 185-199.

Mackinnon, M.J., Georges, M.A. (1992). The effects of selection on linkage analysis for quantitative traits. Genetics, 132, 1177-1185.

Marron, N., Delay, D., Petit, J.M., Dreyer, E., Kahlem, G., Delmotte, F.M., Brignolas, F. (2002). Physiological traits of two Populus X euramericana clones, Luisa Avanzo and Dorskamp, during a water stress and re-watering cycle. Tree Physiol., 22, 849- 858.

O'Toole, J.C. (1982). In "Drought Resistance in Crops with Emphasis on Rice." (pp. 195-213). IRRI.

O’Toole, J.C. (1989). Breeding for drought resistance in cereals, Emerging new technologies. In Baker FWG (ed) Drought resistance in cereals, (pp. 81-94). UK: CAB international Wallingford.

Peng, J., Richards, D.E., Hartley, N.M., Murphy, G.P., Devos, K.M., Flintham, J.E., Beales, J., Fish, L.J., Worland, A.J., Pelica, F., Sudhakar, D., Christou, P., Snape, J.W., Gale, M.D., Harberd, N.P. (1999). Green revolution' genes encode mutant gibberellin response modulators. Nature, 400, 256- 261.

Seyfu, K. (1993). Tef (Eragrostistef): breeding, genetic resources, agronomy, utilization and role in Ethiopian agriculture. Institute of Agricultural Research, Addis Abeba, Ethiopia.

Seyfu, K. (1997). Tef (Eragrostis tef (Zucc.)Trotter. Promoting the conservation and use of underutilized and neglected crops. (pp12.) Institute of Plant Genetics and Crop Plant Research, Gatersleben/International Plant Genetic Resources institute, Rome: Italy.

Tanksley, S.D. (1993). Mapping polygenes. Annu. Rev. Genet., 27,205- 233.

Tavasoli, A. (1986). The cytology of Eragrostis with special reference to E. tef and its relatives. Ph D thesis, Royal Holloway College, University of London, UK.

Tefera, H., Assefa, K., Belay, G. (2003). Evaluation of interspecific recombinant inbred lines of Eragrostistef x E. pilosa. J. Genet. Breed., 57, 21-30.

Vamerali, T., Saccomani, M., Bona, S., Mosca, G., Guarise, M. and Ganis, A. (2003). A comparison of root characteristics in relation to nutrient and water stress in two maize hybrids. Plant Soil, 255,157-167.

Wang, D.L., Zhu, J., Li, Z.K., Paterson, A. (1999). Mapping QTLs with epistatic and QTL X environmental interactions by mixed model approaches. Theor. Appl. Genet., 99, 1255-1264.

Wang, S., Basten, C.J., Zeng, Z.B. (2006). Windows QTL Cartographer 2.5. Department of Statistics, North Carolina State University, Raleigh, NC [online] (http://statgen.ncsu.edu/qtlcart/WQTLCart.htm.)

Watson, L., Dallwitz, M.J. (1992). The Grass Genera of the World, ed. (pp 1). CAB International: Wallingford.

Whalley, W.R., Clark, L.J., Growing, D.G., Cope, R.E., Lodge, R.J., Leeds-Harrison, P.B. (2005). Does soil strength play a role in wheat yield losses caused by soil drying? Plant and Soil, 280, 279- 290.

$\mathrm{Wu}, \mathrm{Y}$., Cosgrove, D.J. (2000). Adaptation of roots to low water potentials by changes in cell wall extensibility and cell wall proteins. J. Exp. Bot., 51, 1543-1553. 
Yu, J., Ramesh, K.V., Elizabeth, G., Benscher, D., Hailu, T., Sorrells, M.E. (2006). A genetic linkage map for tef [Eragrostis tef (Zucc.) Trotter]. Theor. Appl. Genet. 113, 1093-1102.

Yu, J., Ramesh, K.V., Elizabeth, G., Benscher, D., Hailu, T., Sorrells, M.E. (2007). QTL mapping of agronomic traits in tef [Eragrostis tef (Zucc) Trotter]. BMC Plant Biology, 7, 30.

Zeng, B.Z. (1994). Precision mapping of quantitative trait loci. Genetics, 136, 1457-1468.

Zhang, W.P., Shen, X.Y., Wu, S.P., Hu, B., Liao, C.Y. (2001). QTLs and epistasis for seminal root length under a different water supply in rice. Theor. Appl. Genet., 103,118-123.

Table 1. Water potential and water contents of the soil. Soil samples were harvested and their respective water status was measured before (initial) and after (final) the experiment. The presented data represent the mean \pm S.E $(\mathrm{n}=20)$ of one root box

\begin{tabular}{|c|c|c|cc|}
\hline \multirow{2}{*}{ Treatments } & \multicolumn{2}{|c|}{ Water potential (MPa) } & \multicolumn{2}{c|}{ Soil water contents (W/W)in \% } \\
\cline { 2 - 5 } & Initial & Final & Initial & Final \\
\hline Well-watered & $0.2 \pm 0.11$ & $0.2 \pm 0.30$ & $35.0 \pm 0.02$ & $37.0 \pm 0.02$ \\
\hline Water-stressed & $1.6 \pm 0.60$ & $8.5 \pm 1.60$ & $7.6 \pm 0.11$ & $2.0 \pm 0.20$ \\
\hline
\end{tabular}

Table 2. Simple correlations between mean plant height and primary root length of 94 RILs under well-watered and water stressed conditions after 15 days of culturing were estimated. ${ }^{* *}$ Significant at the $\mathrm{P}<1 \%$ level; * Significant at the $\mathrm{P}<5 \%$ level.

\begin{tabular}{|c|c|c|}
\hline Variables & PHC & PHS \\
\hline PRLC & $-0.43^{* *}$ & \\
\hline PRLS & & $-0.24 *$ \\
\hline
\end{tabular}


Table 3. QTLs for PH and PRL. QTLs for plant height under well- watered (PHC) and water- stressed (PHS), primary root length under well- watered (PRLC), water- stressed (PRLS) and differential value between stressed and irrigated conditions (PRLD) at linkage group (LG) to the nearest flanking markers $(P<0.05)$ were listed. Effects of substituting a single allele from one parent to another (A) and phenotypic variation explained by a single QTL $\left(\mathrm{R}^{2}\right)$ for the six traits were evaluated with the RILs. P indicates probability that the putative QTL is unlinked to the nearest marker by a single marker analysis method. The - value indicates allele from E.pilosa. $\left({ }^{*}\right)$ Locus was specified with flanking markers or a marker when it located within $3 \mathrm{cM}$. (†) phenotypic variation explained by all markers identified for a trait and determined by multiple interval mapping.

\begin{tabular}{|c|c|c|c|c|c|c|c|c|c|c|}
\hline \multirow[b]{2}{*}{ Trait } & \multirow[b]{2}{*}{ LG } & \multirow[b]{2}{*}{ Locus(*) } & \multirow[b]{2}{*}{ LOD } & \multicolumn{4}{|c|}{$\mathrm{IM}$} & \multicolumn{3}{|c|}{ CIM } \\
\hline & & & & $\mathrm{P}$ & LOD & A & $\mathrm{R}^{2} \%$ & LOD & A & $\mathrm{R}^{2} \%$ \\
\hline \multirow[t]{6}{*}{$\mathrm{PHC}$} & 1 & TCD99b / CNLT119 & 3.9 & 0.00 & 5.0 & 1.70 & 13 & 5.4 & 1.7 & 16 \\
\hline & 5 & RZ448 / TCD230c & 3.9 & 0.02 & 4.0 & 0.06 & 2 & 4.4 & 0.4 & 2 \\
\hline & 6 & CNLT49b / ISSR548b & 3.9 & 0.00 & 3.2 & -0.06 & 3 & 3.9 & -0.1 & 3 \\
\hline & 9 & CNLT62 & 3.9 & 0.00 & 4.2 & -0.08 & 2 & 4.6 & -0.1 & 2 \\
\hline & 17 & CNLT149/R7214b & 3.9 & 0.01 & 15.5 & 0.09 & 7 & 16.6 & 0.1 & 7 \\
\hline & & 員otal & & & & & & & & 20 \\
\hline \multirow[t]{8}{*}{ PHS } & 1 & RZ154 & 3.3 & 0.04 & 9.3 & 1.9 & 18 & 10.4 & 1.9 & 20 \\
\hline & 2 & TCD273 & 3.3 & 0.02 & 3.2 & 0.86 & 11 & 4.8 & 0.8 & 12 \\
\hline & 3 & TCD35 & 3.3 & 0.00 & 4.1 & 0.68 & 10 & 3.5 & 0.5 & 12 \\
\hline & 6 & RM170b / RM176 & 3.3 & 0.03 & 3.1 & -0.78 & 3 & 5.1 & -0.8 & 3 \\
\hline & 8 & ISSR548a & 3.3 & 0.03 & 3.3 & 0.51 & 2 & 4.1 & 0.4 & 2 \\
\hline & 11 & ISSR842e / RZ166 & 3.3 & 0.01 & 3.3 & 0.78 & 4 & 3.5 & 0.8 & \\
\hline & 13 & RM124c / RZ467a & 3.3 & 0.03 & 3.3 & 0.62 & 2 & 3.3 & 0.7 & 2 \\
\hline & & Total & & & & & & & & 44 \\
\hline \multirow[t]{12}{*}{ PHD } & 1 & RZ154 / TCD230a & 3.5 & 0.00 & 3.5 & 0.60 & 18 & 3.5 & 0.6 & 19 \\
\hline & 4 & RZ962b & 3.5 & 0.03 & 5.3 & 0.80 & 3 & 6.0 & 0.8 & 3 \\
\hline & 6 & CNLT49b & 3.5 & 0.00 & 25 & -1.50 & 11 & 25.8 & -1.5 & 12 \\
\hline & 7 & CNLT145 / RZ698a & 3.5 & 0.02 & 6.2 & -0.80 & 7 & 7.1 & -1.3 & \\
\hline & 7 & FM41 / TCD230b & 3.5 & 0.03 & 4 & -0.70 & 2 & 4.1 & -0.8 & 3 \\
\hline & 10 & ISSR842c & 3.5 & 0.03 & 5.1 & -0.70 & 2 & 5.3 & -0.6 & 2 \\
\hline & 12 & RZ962a & 3.5 & 0.03 & 3.2 & -0.80 & 3 & 3.6 & -0.8 & 3 \\
\hline & & Total & & & & & & & & 38 \\
\hline & 2 & ISSR842g & 3.2 & 0.03 & 6.2 & -1.50 & 3 & 6.7 & -1.0 & 3 \\
\hline & 2 & RZ962c & 3.2 & 0.04 & 3.2 & 0.90 & 2 & 3.7 & 0.9 & 2 \\
\hline & 2 & RM106 / TCD273 & 3.2 & 0.03 & 4.2 & 1.10 & 3 & 4.6 & 1.1 & 3 \\
\hline & 6 & CNLT49b & 3.2 & 0.01 & 5.2 & 0.60 & 15 & 5.3 & 0.7 & 16 \\
\hline \multirow[t]{8}{*}{ PRLC } & 6 & ISSR548b & 3.2 & 0.03 & 4.0 & 1.40 & 3 & 4.2 & 1.3 & 3 \\
\hline & 6 & RM170b & 3.2 & 0.03 & 17.8 & -1.90 & 8 & 18.1 & -2.0 & 9 \\
\hline & 10 & TCD327b & 3.2 & 0.00 & 5.3 & -1.20 & 9 & 5.3 & -1.1 & 10 \\
\hline & 12 & TCD205 / TCD5 & 3.2 & 0.02 & 5.2 & 0.80 & 13 & 5.8 & 0.8 & 15 \\
\hline & 15 & CNLT137-T13 / CNLT154 & 3.2 & 0.02 & 3.2 & -1.00 & 2 & 3.5 & -1.1 & 2 \\
\hline & & Total & & & & & & & & 45 \\
\hline & 3 & ISSR549a / CSU38 & 3.2 & 0.02 & 3.2 & 1.10 & 7 & 3.4 & 1.1 & 9 \\
\hline & 6 & CNLT49b & 3.2 & 0.02 & 10.3 & -1.50 & 6 & 13.4 & -1.5 & ( \\
\hline \multirow[t]{4}{*}{ PRLS } & 6 & RM170b / RM176 & 3.2 & 0.03 & 6.0 & -1.00 & 3 & 6.3 & -1.0 & \\
\hline & 10 & CNLT78 / CNLT41a & 3.2 & 0.02 & 11.2 & -2.10 & 7 & 12.6 & 1.9 & \\
\hline & 10 & ISSR842c & 3.2 & 0.02 & 7.5 & -1.30 & 2 & 8 & -1.1 & \\
\hline & 11 & DuPw4 / ISSR842e & 3.2 & 0.02 & 4.1 & 1.20 & 4 & 5.3 & 1.2 & \\
\hline
\end{tabular}


Table 4. Epistatic effect conferred from the combination of loci. Epistatic loci for plant height under well-watered (SHC) and water-stressed (SHS) conditions, and primary root length under well-watered (PRC), water-stressed (PRLS) conditions were calculated with the QTL mapper method.

\begin{tabular}{|c|c|c|c|c|c|c|c|}
\hline \multirow[t]{2}{*}{ Traits } & \multicolumn{3}{|c|}{ Marker 1} & \multicolumn{4}{|l|}{ Marker 2} \\
\hline & $\mathrm{LG}^{\mathrm{a}}$ & $\begin{array}{c}\mathrm{L} \\
\text { ocus }\end{array}$ & $\mathrm{LG}^{\mathrm{a}}$ & Locus & LOD $^{b}$ & $A^{c}$ & $\mathrm{R}^{2 \mathrm{~d}} \%$ \\
\hline \multirow{2}{*}{ PHC } & 3 & TCD95 & 6 & CNLT49b & 5.0 & -1.0 & 14 \\
\hline & 7 & ISSR840a & 11 & ISSR842e & 5.3 & -1.1 & 18 \\
\hline \multirow{2}{*}{ PHS } & 6 & RM170b & 3 & TCD95 & 3.1 & 0.9 & 11 \\
\hline & 3 & TCD95 & 8 & TCD227a & 5.1 & 0.7 & 5 \\
\hline PRLC & 3 & TCD95 & 6 & RM170b & 4.3 & 2.2 & 20 \\
\hline PRLS & 6 & RM170b & 8 & CDO38 & 4.2 & -2.1 & 18 \\
\hline \multirow[t]{2}{*}{ Traits } & \multicolumn{3}{|c|}{ Marker 1} & \multicolumn{4}{|l|}{ Marker 2} \\
\hline & $L G^{a}$ & $\begin{array}{c}\mathrm{L} \\
\text { ocus }\end{array}$ & $\mathrm{LG}^{\mathrm{a}}$ & Locus & $\mathrm{LOD}^{\mathrm{b}}$ & $A^{c}$ & $\mathrm{R}^{2 \mathrm{~d}} \%$ \\
\hline \multirow{2}{*}{ PHC } & 3 & TCD95 & 6 & CNLT49b & 5.0 & -1.0 & 14 \\
\hline & 7 & ISSR840a & 11 & ISSR842e & 5.3 & -1.1 & 18 \\
\hline \multirow{2}{*}{ PHS } & 6 & RM170b & 3 & TCD95 & 3.1 & 0.9 & 11 \\
\hline & 3 & TCD95 & 8 & TCD227a & 5.1 & 0.7 & 5 \\
\hline PRLC & 3 & TCD95 & 6 & RM170b & 4.3 & 2.2 & 20 \\
\hline PRLS & 6 & RM170b & 8 & CDO38 & 4.2 & -2.1 & 18 \\
\hline
\end{tabular}

${ }^{a}$ Linkage group

${ }^{\mathrm{b}}$ LOD score calculated by QTL mapper 2.0 at $P<0.05$ level of probability.

${ }^{\mathrm{c}}$ Additive effect.

${ }^{\mathrm{d}}$ Percentage of the phenotypic variance explained by the marker genotypes at the locus. 

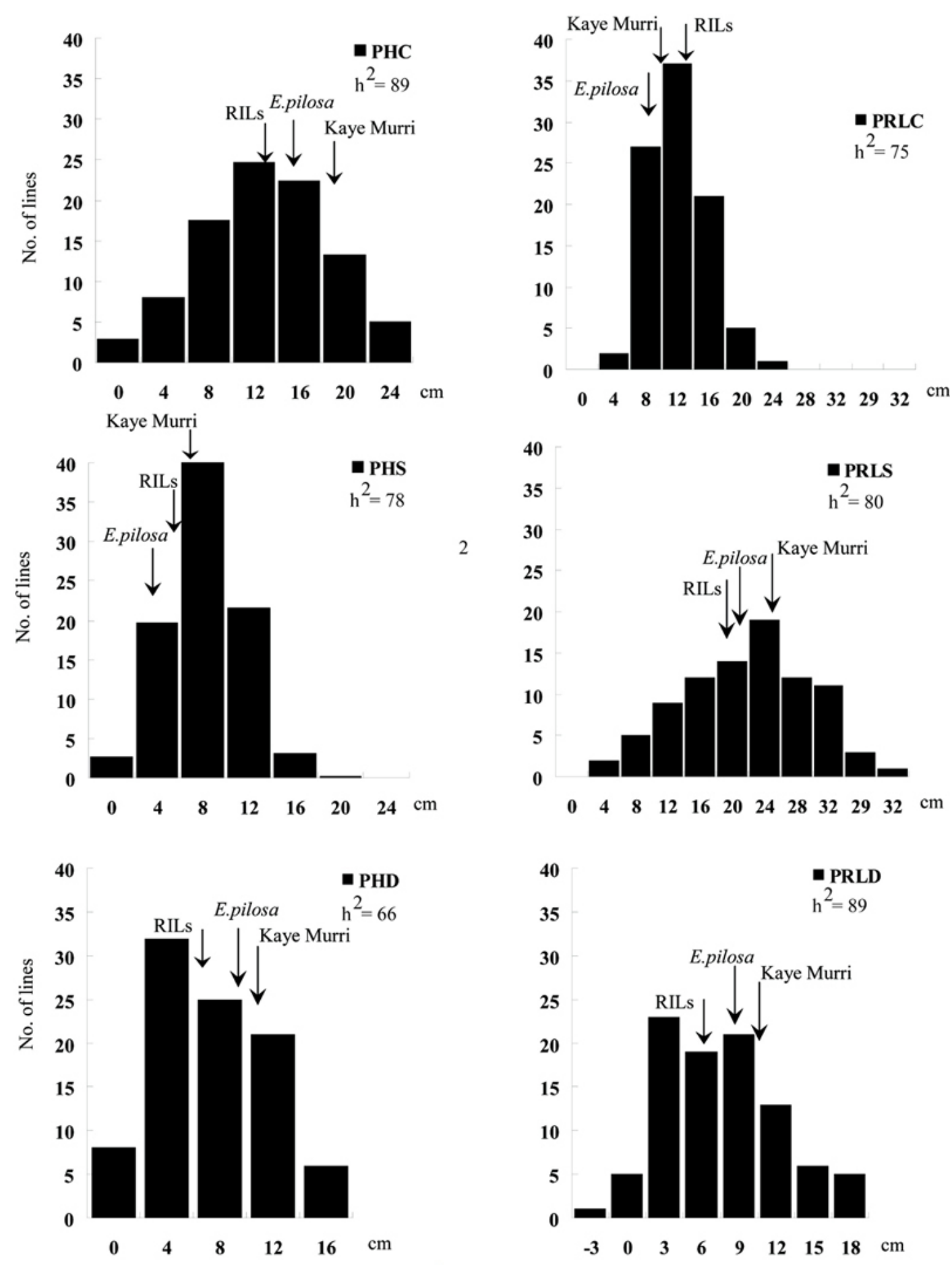

Figure 1. Frequency distribution for the sizes of plants of the RILs cultured under well- watered or waterstressed conditions. Plant height under- well watered (PHC) and water- stressed (PHS), primary root length under well- watered (PRLC) and water- stressed (PRLS) and differential value of plant height (PHD) and primary root length (PRLD) between well watered and water stressed conditions were measured after 15 days of culturing. Data is the mean, $n=10 / 10$ root box. 10 plants were cultured in one box. 

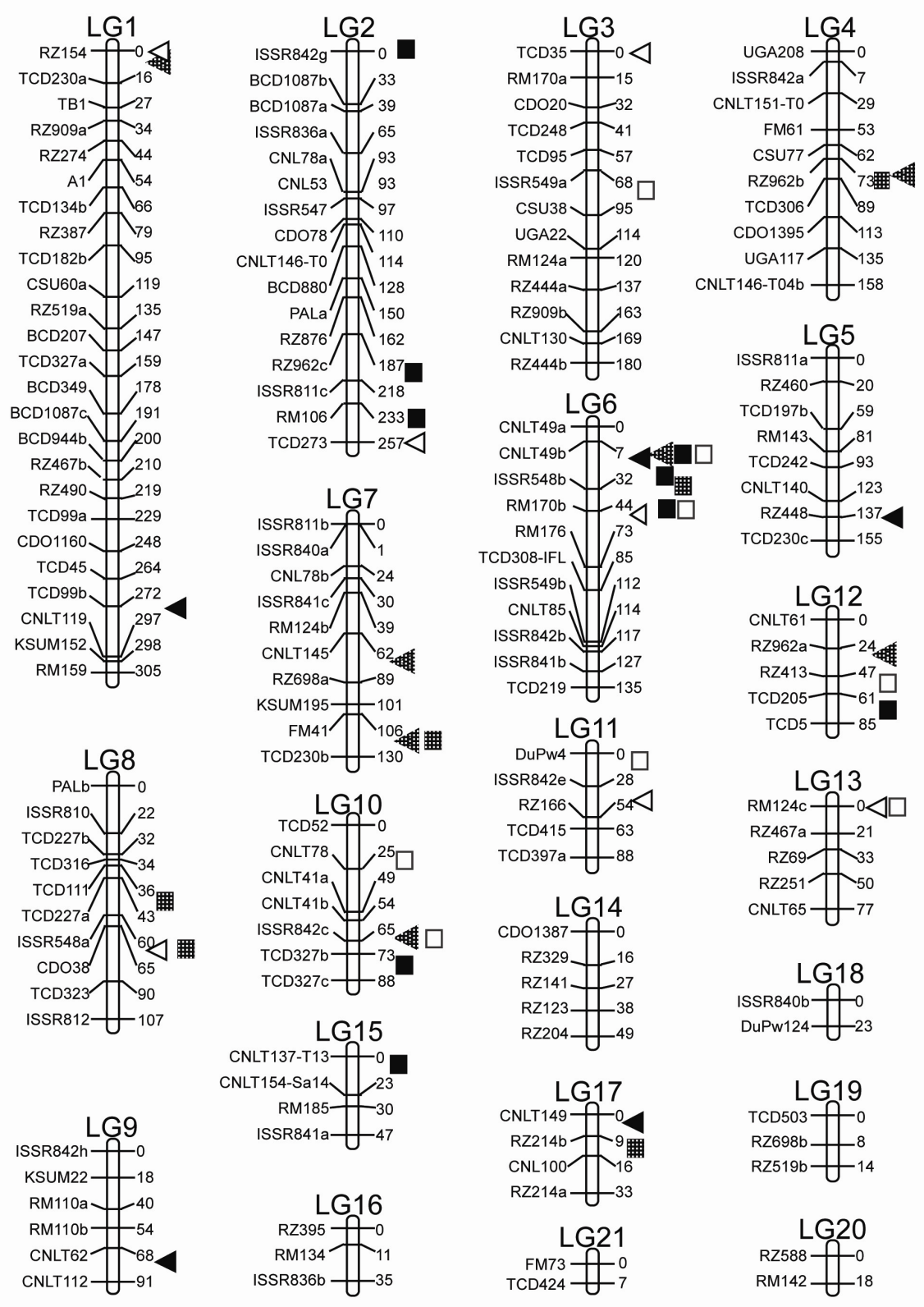

4 :PHC $\triangleleft$ :PHS

唯:PHD

:PRLC $\square: P R L S \quad$ 糟:PRLD

Figure 2. QTLs of plant size of tef. QTLs for plant height under well- watered conditions (PHC) and under water- stressed conditions (PHS), primary root under well watered conditions (PRLC) and under water- stressed conditions (PRLS); difference of the plant height and primary root between water- stressed and well- watered conditions (PHD and PRLD) in the RILs population derived from a cross between Kaye Murri and E. Pilosa (305). The designation on the right is the marker name, and on the left is the map distance based on the Kosambi function. 